\title{
Multi-View Whole Lung Radiomics Quantitative Analysis of COVID-19 Pneumonia Based on Machine Learning
}

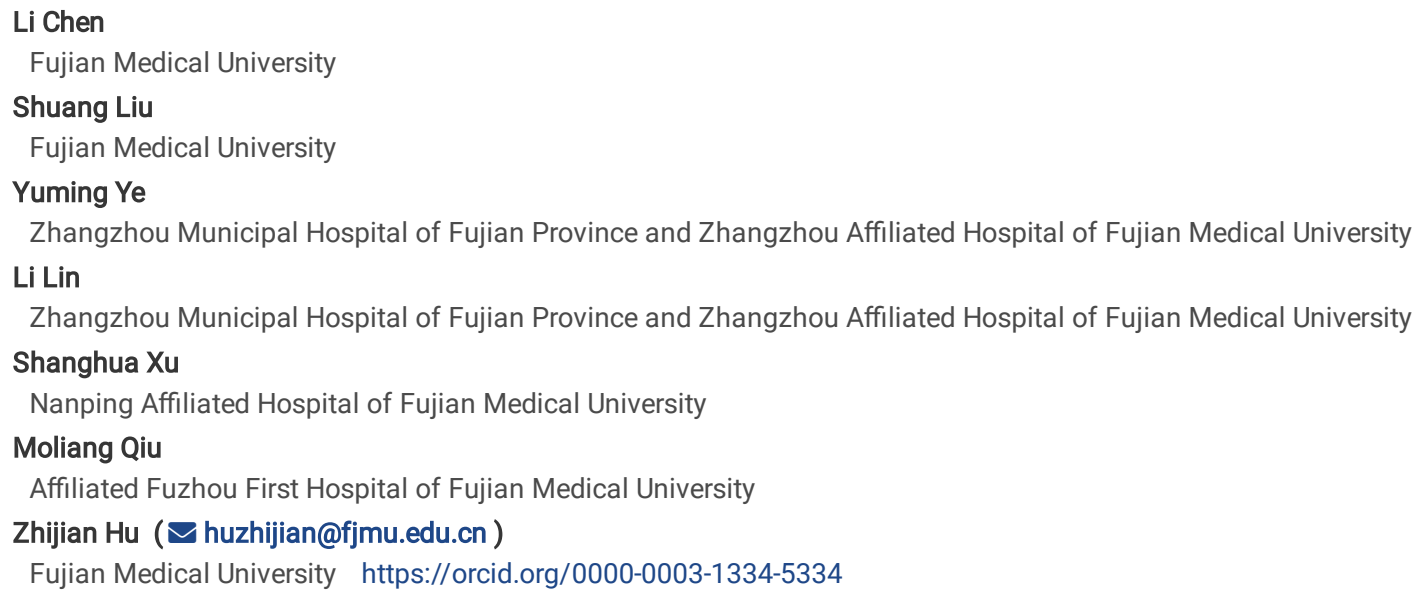




\section{Abstract}

Background: Quantitative and radiomics imaging could realize non-invasive disease diagnosis. This study aimed to evaluate radiomics features of the whole lung for predicting new coronavirus disease 2019(COVID-19) from different views, and to investigate new radiomics features. 75 patients were retrospectively enrolled from December 1, 2019 to December 31, 2020. Both lungs were segmented by an unsupervised hybrid image segmentation approach. Radiomics features of the transverse plane, coronal plane and sagittal plane were separately extracted. After utilizing least absolute shrinkage and selection operator (LASSO), three radiomics models based on key radiomics features were built by machine learning. Meanwhile, the different categories radiomics models were constructed by the particle swarm optimization-deep extreme learning machine(PSO-DELM). Predictive accuracy, sensitivity, specificity and areas under receiver operating characteristic curve (AUC) were evaluated performances of these radiomics models.

Results: Training and test cohorts had similar distributions of age and pneumonia type. 13 (transverse plane), 4 (coronal plane) and 8 (sagittal plane) selected features were constructed radiomics models in training cohort. Radiomics models based on PSO-DELM in the transverse plane, coronal plane and sagittal plane showed the favorable performance in the testing cohort(AUC $=0.9444,0.8636$ and 0.9444 , respectively). The phase congruency feature showed the stable predictive performance (AUC>0.9) among these radiomics features on the three different plane.

Conclusions: Multi-view whole lung radiomics features could effectively differentiate COVID-19 from other types of pneumonia. Phase congruency may be attempted as a radiomics biomarker for the identification of pulmonary diseases. Merging the radiomics features into PSO-DELM is a promising direction for future research about medical radiology and deep learning.

\section{Introduction}

With the outbreak of the Corona Virus Disease (COVID-19), it had rapidly spread to China and the rest of the world, and was becoming a worldwide epidemic. It result that more than 12,102,328 confirmed cases of the COVID-19 and more than 551,046 deaths all over world [1]. Of infected individuals, $80 \%$ were asymptomatic or only manifest mild symptoms [2]. CT scans were an effective non-invasive diagnostic method that was proved higher sensitivity than existing reverse transcriptase-polymerase chain reactions (RT-PCR) kits [3]. Patchy ground glass opacities, multiple, bilateral had been advised as the feature of COVID-19 infection on CT images [4-5]. The lesions had mainly a sub pleural distribution, and were extremely similar to other types of pneumonia [6]. However, these radiological findings were a subjective experience evaluation of the clinicians and radiologists. So a rapid quantitative analysis method base on machine learning will contribute to the clinicians and radiologists to diagnose COVID-19.

Radiomics contained the high-throughput quantitative CT imaging features and had probable to generate radiomics biomarkers which can accurate diagnosis and treatment[7-9]. There were also varieties of studies reported that it was potential for the radiomics in cancer and tumor to evaluate the resectability, treatment response, and prognosis [10-15]. Radiomics features were the useful diagnostic biomarkers that could afford accurate quantitative information for clinical data [16]. It was a first step in the development of radiomics combined with intelligence algorithms. So radiomics features might afford a effective tool to diagnosis of COVID-19[17, 18]. Basic CT features had been proved useful for diagnosing COVID-19, but it was needed to develop to utilize CT radiomics biomarkers as predictive biomarkers for distinguishing COVID-19. There were most literatures reported that had using a CT radiomic model to distinguish COVID-19 based on transverse plane. The lesions were segmented by using professional software or manually delineated by radiologists.[17, 18]. It was very labor-intensive and time-consuming. Currently, there is fewer reported research on the radiomics features of the whole-lung which were automatically segmented based on multiple views. The distribution of ground glass opacities, number of lesions, maximum lesion range, and lobe involvement can be observed not only in the transverse plane of lung, but also in coronal plane and sagittal plane. So radiomics features of different planes could reflect richer radiomics information for radiologists.

Therefore, the aim of this study were (1)to inquire into and quantitatively analyze multi-view whole lung radiomics in differentiating COVID-19 from other types of pneumonia by using machine learning methods and(2) to discover new radiomics biomarkers correlated with COVID-19, and (3) to assess the predictive ability of radiomis models before diagnosis and individual treatment.

\section{Materials And Methods}

\section{Data}

75 patients with pathologically confirmed between December 2019 and February 2020 were enrolled in this study. All patients from three hospitals underwent low-dose CT scans. The exclusion criteria were met by COVID-19 patients with (1) lung parenchyma was inconspicuous or too small that could not be identified by CT; (2) clinic pathological data were shortage; (3) large interval time between CT examination and pathological diagnosis was greater than 1 week. The inclusion criteria were met by other types of pneumonia patients with common pneumonias (e.g. bacterial pneumonia) before treatment. To make the model more generalizable and robust, 40 patients (20 COVID-19 patients/20 other types of pneumonia patients) from Zhangzhou Affiliated of Fujian Medical University were enrolled in training cohort. 19 patients from Nanpin Affiliated of Fujian Medical University (10 COVID-19 patients/8 other types of pneumonia patients) were enrolled in validation cohort. 9 COVID-19 patients from Nanpin Affiliated of Fujian Medical University and 8 other types of pneumonia patients from Affiliated Fuzhou First Hospital of Fujian Medical University were alone enrolled in test cohort. The CT scans were acquired from Siemens (SOMOTOM Definition Flash, Erlangen, Germany); further details regarding image acquisition were presented in section 1 of Appendix A1.

\section{Image segmentation and feature extraction}

All patients' lung pulmonary windows images were manually separated into transverse planes, coronal planes and sagittal planes. To reduce the spending of manual segmentation lesions, the whole lung as region of interest (ROI) was segmented automatically based on a unsupervised hybrid approach of fuzzy c- 
means and gray-level co-occurrence matrix (Fig.1). Segmentation ROI method was explained in detail in section 2 of Appendix A1 and it was performed through MATLAB software(version 2020a; The MathWorks Inc., Natick, MA, USA). The two radiologists assessed the results of segmentation and reached a consensus by discussion.

210 radiomics features were separately extracted form $\mathrm{ROI}$ of the transverse plane, coronal plane and sagittal plane. These features were divided into 10 categories:(a) Hu invariant moment(HU), (b) Gray-level co-occurrence matrix(GLCM),(c)Local entropy,(d) Statistics features including shape, sizes, extremes and so on, (e) Gray-level run-length matrix (GLRLM), (f)Entropy, (g)Gabor wavelet filter(Gabor), (h)Hessian matrix (Hessian), (i) Histogram of Oriented Gradients (HOG), (j)Phase congruency(Phase). The process of feature extraction was described in detail in section 3 of Appendix A1. This process was performed by using MATLAB software.

\section{Radiomics signature construction}

High-dimensional data were appropriate to use the least absolute shrinkage and selection operator (LASSO) method to reduce the dimension and this method was usually utilized as extracting the optimal features in other radiomics studies of the pneumonia[18-19]. The optimal features were selected from the radiomics features on the training cohort of three different planes. The "glmnet" package in R software (v3.5.3; R Development Core Team) was employed for LASSO logistic regression.

\section{Construction of multiple radiomics predictive models}

We validated and evaluated the performances of radiomics biomarkers on the validation and test cohorts from three different views. The optimal radiomics features were separately constructed radiomics models by using the support vector machine(SVM), particle swarm optimization-deep extreme learning machine(PSO-DELM)[20], back propagation-neural network (BP) in training cohort. To discover and investigate the function of different category radiomics features, we also constructed different category radiomics models and analyzed the predicting performance of these features in the three planes. The parameter settings were optimized and adjusted in the validation cohort and presented in section 4 of Appendix A1. The predictive accuracy, sensitivity, specificity, the area under receiver operating characteristic curve (AUC) were evaluated the performance of radiomics models. The processes were executed in Matlab 2020a.

\section{Statistical analysis}

Statistical analysis was implemented using R (version 3.5.3). Continuous variables were expressed as mean \pm standard deviation or median and interquartile range (IQR) according the results of normality test, whereas categorical variables were expressed as absolute number and percentage. Chi-square test was used to compare the differences in patients' clinical factors (categorical variables) among two cohorts. Independent sample t-tests or Wilcoxon test were used to compare patients' clinical factors (continuous variables) and various radiomics features among two groups. A two-sided P value $<0.05$ was considered as statistical significance.

\section{Results}

\section{Clinical characteristics of the patients}

The characteristics of training and validation cohorts were shown in Table 1. The median (IQR) ages of the two cohorts were 40.5(32.0-51.0) years and 42.0 (35.8-56.3) years. Training and validation cohorts had similar distributions of age and pneumonia type $(P>0.05)$.

Table 1

Characteristics of patients in the training and validation cohorts

\begin{tabular}{|llllll|}
\hline Variable & Total & Training cohort & Validation cohort & Z/ $\chi^{2}$ & $P$ \\
\hline Age(years) & $41.5(32.8,51.5)$ & $40.5(32.0,51.0)$ & $42.0(35.8,56.3)$ & -0.850 & 0.396 \\
\hline Gender (\%) & & & & 4.329 & 0.036 \\
\hline Male & $30(51.7)$ & $17(42.5)$ & $13(72.2)$ & & \\
\hline Female & $28(48.3)$ & $23(57.5)$ & $5(27.8)$ & & \\
\hline Pneumonia type(\%) & & & & 0.153 & 0.695 \\
\hline CoVID-19 & $30(51.7)$ & $20(50.0)$ & $10(55.6)$ & & \\
\hline other types & $28(48.3)$ & $20(50.0)$ & $8(44.4)$ & & \\
\hline
\end{tabular}

\section{Feature Selection And Radiomics Biomarkers Building}

13 features (transverse plane), 4 features (coronal plane), 8 features (sagittal plane) were respectively selected by LASSO with 10-fold cross test (Fig. 2). We used the optimized lambda to select key features with non-zero coefficients from the coefficient profiles based on 1000 iterations. The optimized lambdas were 0.0095 (transverse plane), 0.1067 (coronal plane), and 0.01146 (sagittal plane), separately. These radiomics features were detailed in Table 2. 
Table 2

Comparison of the radiomics signatures from three views between COVID-19 and other types of pneumonia

\begin{tabular}{|c|c|c|c|}
\hline View & Name & Categories Features & P-value \\
\hline \multirow[t]{13}{*}{ Transverse plane } & hu2 & $\mathrm{HU}$ & $<0.001$ \\
\hline & GLCM_Information measure of correlation1 & GLCM & 0.02 \\
\hline & GLCM_Inverse difference (INV) is homom & GLCM & 0.002 \\
\hline & gabor3 & Gabor & 0.171 \\
\hline & hessian_hist7 & Hessian & 0.554 \\
\hline & hessian_hist11 & Hessian & 0.102 \\
\hline & phasecong_hist18 & Phase congruency & 0.029 \\
\hline & mean_localentropy & Local entropy & 0.071 \\
\hline & locent9 & Local entropy & 0.027 \\
\hline & locent18 & Local entropy & 0.066 \\
\hline & HOG_hist12 & HOG & 0.72 \\
\hline & HOG_hist15 & HOG & 0.418 \\
\hline & HOG_hist17 & HOG & 0.269 \\
\hline \multirow[t]{8}{*}{ Sagittal plane } & stats_MinorAxisLength & Statistics & 0.002 \\
\hline & stats_ThinnessRatio & Statistics & 0.856 \\
\hline & max_hessiandet & Hessian matrix & 0.387 \\
\hline & hessian_hist10 & Hessian matrix & 0.129 \\
\hline & locent6 & Local entropy & 0.036 \\
\hline & HOG_hist9 & HOG & $<0.001$ \\
\hline & HOG_hist15 & HOG & 0.071 \\
\hline & HOG_hist18 & HOG & 0.048 \\
\hline \multirow[t]{4}{*}{ Coronal plane } & GLCM_Sum of sqaures: Variance & GLCM & 0.154 \\
\hline & stats_Extrema7 & Statistics & 0.226 \\
\hline & min_hessiandet & Hessian matrix & $<0.001$ \\
\hline & phasecong_hist12 & Phase congruency & 0.638 \\
\hline
\end{tabular}

\section{Predictive Ability Of Radiomics Models}

The optimal features were implemented to generate the radiomics model for distinguishing COVID-19. We built and evaluated three radiomics models for selecting the best model; the performances of these models were described by accuracy, sensitivity, specificity, AUC as shown in Table 3. To develop and investigate the different category radiomics models on the different planes, we utilized PSO-DELM models to construct and validate the predictive ability of the different categories radiomics features, that were shown in Table 4 and Fig. 3. 
Table 3

Predictive ability of three machine learning models in the three cohorts.

\begin{tabular}{|c|c|c|c|c|c|c|c|c|c|c|c|c|}
\hline \multirow[t]{2}{*}{ View } & \multirow[t]{2}{*}{ Model } & \multicolumn{4}{|c|}{ Training cohort } & \multicolumn{4}{|c|}{ Validation cohort } & \multicolumn{3}{|l|}{ test cohort } \\
\hline & & Accuracy & Sensitivity & Specificity & AUC & Accuracy & Sensitivity & Specificity & AUC & Accuracy & Sensitivity & Spec \\
\hline \multirow{3}{*}{$\begin{array}{l}\text { Transverse } \\
\text { plane }\end{array}$} & SVM & 1.0000 & 1.0000 & 1.0000 & 1.0000 & 0.3889 & 0 & 0.8750 & 0.4375 & 0.5294 & 0.1111 & 1.00 \\
\hline & $\mathrm{BP}$ & 1.0000 & 1.0000 & 1.0000 & 1.0000 & 0.9444 & 1.0000 & 0.8750 & 0.9375 & 0.9412 & 0.8889 & 1.00 \\
\hline & $\begin{array}{l}\text { PSO- } \\
\text { DELM }\end{array}$ & 1.0000 & 1.0000 & 1.0000 & 1.0000 & 0.8333 & 0.9000 & 0.7500 & 0.8377 & 0.9412 & 0.8889 & 1.00 \\
\hline \multirow{3}{*}{$\begin{array}{l}\text { Coronal } \\
\text { plane }\end{array}$} & SVM & 1.0000 & 1.0000 & 0.7500 & 0.8750 & 0.6667 & 1.0000 & 0 & 0.5000 & 0.5294 & 1.0000 & 0 \\
\hline & $\mathrm{BP}$ & 1.0000 & 1.0000 & 1.0000 & 1.0000 & 0.9333 & 1.0000 & 0.8000 & 0.9000 & 0.8235 & 0.6667 & 1.001 \\
\hline & $\begin{array}{l}\text { PSO- } \\
\text { DELM }\end{array}$ & 0.8636 & 1.0000 & 0.2500 & 0.9286 & 0.8000 & 0.9000 & 0.6000 & 0.7841 & 0.8235 & 0.6667 & 1.00 \\
\hline \multirow{3}{*}{$\begin{array}{l}\text { Sagittal } \\
\text { plane }\end{array}$} & SVM & 0.8500 & 1.0000 & 0.2500 & 0.6250 & 0.7273 & 0.7000 & 1.0000 & 0.8500 & 0.8235 & 0.6667 & 1.001 \\
\hline & $\mathrm{BP}$ & 1.0000 & 1.0000 & 1.0000 & 1.0000 & 0.9091 & 0.9000 & 1.0000 & 0.9500 & 0.9412 & 1.0000 & $0.87 !$ \\
\hline & $\begin{array}{l}\text { PSO- } \\
\text { DELM }\end{array}$ & 0.9500 & 1.0000 & 0.7500 & 0.9706 & 0.9091 & 0.9000 & 1.0000 & 0.7500 & 0.9412 & 0.8889 & 1.00 \\
\hline
\end{tabular}

Note. AUC(area under the receiver operating characteristic curve) were values and were calculated by applying confusion matrix. Models: SVM = support vect = back propagation-neural network, PSO-DELM = particle swarm optimization- deep extreme learning machine 
Table 4

The performances of the different categories radiomics features in the transverse plane, coronal plane and sagittal plane based on the PSO-DELM models

\begin{tabular}{|c|c|c|c|c|c|c|c|c|c|c|}
\hline \multirow[t]{2}{*}{ Views } & \multirow[t]{2}{*}{ Categories } & \multicolumn{3}{|c|}{ Training cohort } & \multicolumn{3}{|c|}{ Validation cohort } & \multicolumn{3}{|c|}{ Test cohort } \\
\hline & & Accuracy & Sensitivity & Specificity & Accuracy & Sensitivity & Specificity & Accuracy & Sensitivity & Specificity \\
\hline Transverse & $\mathrm{HU}$ & 0.8500 & 0.9500 & 0.7500 & 0.8333 & 0.9000 & 0.7500 & 0.4706 & 0.7778 & 0.1250 \\
\hline \multirow[t]{9}{*}{ Plane } & GLCM & 0.7750 & 0.7500 & 0.8000 & 0.7778 & 0.9000 & 0.6250 & 0.8824 & 0.8889 & 0.8750 \\
\hline & Statistics & 0.8000 & 0.8500 & 0.7500 & 0.8889 & 1.0000 & 0.7500 & 0.9412 & 0.8889 & 1.0000 \\
\hline & Gabor & 0.6250 & 0.6000 & 0.6500 & 0.7222 & 0.6000 & 0.8750 & 0.7059 & 0.4444 & 1.0000 \\
\hline & Hessian & 1.0000 & 1.0000 & 1.0000 & 0.9444 & 0.9000 & 1.0000 & 0.9412 & 0.8889 & 1.0000 \\
\hline & Phase & 0.6500 & 0.7000 & 0.6000 & 0.8333 & 1.0000 & 0.6250 & 0.9412 & 0.8889 & 1.0000 \\
\hline & Locent & 0.6500 & 0.9500 & 0.3500 & 0.2222 & 0.2000 & 0.2500 & 0.5294 & 0.1111 & 1.0000 \\
\hline & Entropy & 1.0000 & 1.0000 & 1.0000 & 0.9444 & 1.0000 & 0.8750 & 0.9412 & 0.8889 & 1.0000 \\
\hline & HOG & 1.0000 & 1.0000 & 1.0000 & 0.8889 & 1.0000 & 0.7500 & 0.8824 & 0.7778 & 1.0000 \\
\hline & GLRLM & 1.0000 & 1.0000 & 1.0000 & 0.7778 & 0.8000 & 0.7500 & 0.9412 & 0.8889 & 1.0000 \\
\hline \multirow{10}{*}{$\begin{array}{l}\text { Coronal } \\
\text { Plane }\end{array}$} & $\mathrm{HU}$ & 0.8636 & 1.0000 & 0.2500 & 0.6000 & 0.6000 & 0.6000 & 0.6471 & 0.3333 & 1.0000 \\
\hline & GLCM & 1.0000 & 1.0000 & 1.0000 & 0.8667 & 1.0000 & 0.6000 & 0.9167 & 0.4706 & 0.8889 \\
\hline & Statistics & 1.0000 & 1.0000 & 1.0000 & 0.9333 & 1.0000 & 0.8000 & 0.8235 & 0.6667 & 1.0000 \\
\hline & Gabor & 1.0000 & 1.0000 & 1.0000 & 0.5333 & 0.6000 & 0.4000 & 0.5882 & 0.6667 & 0.5000 \\
\hline & Hessian & 1.0000 & 1.0000 & 1.0000 & 1.0000 & 1.0000 & 1.0000 & 0.9412 & 0.8889 & 1.0000 \\
\hline & Phase & 1.0000 & 1.0000 & 1.0000 & 0.9333 & 1.0000 & 0.8000 & 0.9412 & 0.8889 & 1.0000 \\
\hline & Locent & 0.6364 & 0.6667 & 0.5000 & 0.6667 & 1.0000 & 0 & 0.5882 & 1.0000 & 0.1250 \\
\hline & Entropy & 1.0000 & 1.0000 & 1.0000 & 0.9333 & 1.0000 & 0.8000 & 0.7059 & 1.0000 & 0.3750 \\
\hline & HOG & 1.0000 & 1.0000 & 1.0000 & 0.9333 & 1.0000 & 0.8000 & 0.9412 & 1.0000 & 0.8750 \\
\hline & GLRLM & 1.0000 & 1.0000 & 1.0000 & 0.9333 & 1.0000 & 0.8000 & 0.9412 & 0.8889 & 1.0000 \\
\hline \multirow{10}{*}{$\begin{array}{l}\text { Sagittal } \\
\text { Plane }\end{array}$} & $\mathrm{HU}$ & 0.9500 & 1.0000 & 0.7500 & 0.9091 & 1.0000 & 0.0000 & 0.9412 & 1.0000 & 0.8750 \\
\hline & GLCM & 1.0000 & 1.0000 & 1.0000 & 0.9091 & 1.0000 & 0.0000 & 0.5882 & 1.0000 & 0.1250 \\
\hline & Statistics & 1.0000 & 1.0000 & 1.0000 & 1.0000 & 1.0000 & 1.0000 & 0.9412 & 0.8889 & 1.0000 \\
\hline & Gabor & 0.8000 & 1.0000 & 0.0000 & 1.0000 & 1.0000 & 1.0000 & 0.9412 & 0.8889 & 1.0000 \\
\hline & Hessian & 1.0000 & 1.0000 & 1.0000 & 0.9091 & 1.0000 & 0.0000 & 0.9412 & 0.8889 & 1.0000 \\
\hline & Phase & 1.0000 & 1.0000 & 1.0000 & 1.0000 & 1.0000 & 1.0000 & 0.9412 & 0.8889 & 1.0000 \\
\hline & Locent & 0.7000 & 0.8125 & 0.2500 & 0.4545 & 0.4000 & 1.0000 & 0.1176 & 0.2222 & 0 \\
\hline & Entropy & 1.0000 & 1.0000 & 1.0000 & 0.9091 & 0.9000 & 1.0000 & 0.9412 & 0.8889 & 1.0000 \\
\hline & HOG & 1.0000 & 1.0000 & 1.0000 & 0.9091 & 0.9000 & 1.0000 & 0.9412 & 0.8889 & 1.0000 \\
\hline & GLRLM & 1.0000 & 1.0000 & 1.0000 & 0.8182 & 0.8000 & 1.0000 & 0.9412 & 0.8889 & 1.0000 \\
\hline
\end{tabular}

\section{Discussion}

In our study, we quantitatively analyzed multi-view whole lung radiomics to distinguish COVID-19 from other types of pneumonia by using machine learning methods. The radiomics model based on PSO-DELM could effectively differentiate COVID-19 from other types of pneumonia before diagnosis. Phase congruency features were used as radiomics features at the first time, it may be considered as radiomics biomarkers to predict COVID-19.

The previous studies showed that COVID-19 were significantly associated with the following CT imaging characteristics, such as bilateral lungs, peripheral or diffuse distribution, ground glass opacity, maximum lesion range, number of lesions, lobe involvement, Hilar and mediastinal lymph nodes enlargement, and so on[21-22]. All of these CT characteristics were almost expressed in the lung pulmonary window (except mediastinal lymph nodes enlargement), so the whole lung of different planes could be displayed the richer radiomics information of COVID-19 to radiologists. The current most studies were focused on the radiomics features of the lesions, these lesions were segmented by manual segmentation or by deep learning approaches[17, 21]. Manual segmentation was 
expensive to study and diagnosis in scarcity of clinical resources. Meanwhile, deep learning methods were need a amount of labels and the precision of the segmentation need to be improved(DSI:0.778)[21]. In our study, we tried to segment the lung unsupervised without manual operation or imaging software, the performance of segmentation was as good as classical region growing method(DSI: 0.9230 vs 0.9092) that was detailed in Appendix A1. FANG et al's study[17] segmented the lesion area by using a imaging software, AUC value of radiomics model for differentiating COVID-19 based on SVM was 0.826 . It showed that the radiomics model based on PSO-DELM(AUC 0.9444) was better than radiomics models based on SVM(AUC 0.5556) in our study. For the transverse plane and sagittal plane, the sensitivity of radiomics model based on PSO-DELM was higher than 0.8889, which meant that the whole-lung radiomics features can accurately assess the COVID-19.

Non-invasive disease diagnosis could be realized by using imaging radiomics, $2 \mathrm{D}$ image features previously studied were only extracted from the transverse plane [23]. 2D image radiomics features extracted from the transverse plane or 3D radiomics features extracted from voxel of lesions could not fully describe the whole COVID-19 radiomics features comprehensively, but sagittal plane, coronal plane and transverse plane could roundly contain radiomics information of the lesions (including the shape, size, location). In this study, we found that Hessian matrix features were all captured on the three planes. It indicated that these features can display gray gradient change information from different views. Table 4 showed that the prediction accuracy of these radiomics features achieved 0.9412 both on three different planes. Entropy feature and HOG features which described the shape and texture were captured on the transverse plane and sagittal plane, but the performances of Entropy features were better than HOG features on the transverse plane(Accuracy 0.9412 vs 0.8824 ).

GLCM features and phase congruency features were captured both on the transverse plane and coronal plane, but our study showed that the prediction performance of phase congruency features were better than GLCM features on different planes, that was shown in Table 4 and Fig. 3 . To our knowledge, there were fewer reported researches about phase congruency features used in radiomics. The phase congruency was invariant to image contrast and could extract effective and reliable texture features under different illumination conditions [24]. Phase congruency properly extracted features of any kind of phase angle, it was different from feature detectors based on gradient, which could only extract step features. It was usually used in palm print authentication, face representation technique, image segmentation technique, and so on [24-28]. In our study, phase congruency feature was favorable performance in differentiating COVID-19 from other types of pneumonia on the three planes (all AUC $=0.9444$ ). It may be considered to be as diagnostic biomarker for lung in other diseases.

In this study, we constructed three radiomics models to identifying COVID-19. In the independent test cohort, the prediction accuracy, sensitivity, specificity and AUC of radiomics models based on PSO-DELM showed $0.9412,0.8889,1.0000$ and 0.9444 on the transverse plane, respectively. It also did well on other two planes. The radiomics model based on BP(AUC $=0.9444)$ showed as good as the radiomics model based on PSO-DELM on the transverse plane, but the radiomics model based on SVM performed poorly on all three planes. So the radiomics model based on PSO-DELM could be as a clinical adjunct tool to help radiologists to predict COVID-19 from different views.

There were several limitations of this study. First, it was a retrospective study using a small sample size from three hospitals with no external test data, so small amount of COVID-19 may affect the problems of over-fitting and robustness of the prediction effect. Second, we did not segment the lesions in lung parenchyma that may imply that extracted radiomics features did not express the all radiomics information, because so far there were fewer accurate automatic segmentation technologies. That might affect all real radiomic information. Third, phase congruency feature was used as radiomics features at the first time, but no more proof of availability in other areas of diseases. Besides, 2D radiomics features were only used in our study. 3D radiomics features would improve the predictive ability of our models.

\section{Conclusions}

In conclusion, our study showed that multi-view whole lung radiomics features could effectively predict COVID-19 by using machine learning methods. Phase congruency features were first proposed to use to differentiate COVID-19 from other types of pneumonias. The radiomics models based on PSO-DELM showed favorable performance in predicting COVID-19. We hoped that our first trying of new radiomics features could be helpful for improving the diversification of diagnostic biomarker; employing deep learning to analysis the radiomics features of lung can assist clinical for diagnosis of lung diseases.

\section{Abbreviations}

\section{COVID-19}

new coronavirus disease 2019 pneumonia, AUC = area under the receiver operating characteristic curve, SVM = support vector machine, PSO-DELM = particle swarm optimization-deep extreme learning machine, $\mathrm{BP}=$ back propagation-neural network

\section{Declarations}

Ethical approval and consent to participate This study was approved by the Ethics Committee of Fujian Medical University (Approval No: 202003). Informed consents were obtained from all enrolled patients.

Consent for publicationNot applicable

Availability of data and materials The datasets used and/or analysed during the current study are available from the corresponding author on reasonable request.

Competing interests The authors have no conflicts of interest to declare. 
Funding This work was supported by the Fujian Medical University's Rapid Response Funding Call for COVID-19 Related Research, Fujian, China (Grant No.2020Yj003).

Authors' contributions All authors contributed to the study conception and design. Material preparation, data collection and analysis were performed by Yuming Ye Shanghua Xu, Li Chen, Shuang Liu. The segmentation results of lung were check by Moliang Qiu and Shanghua Xu. The first draft of the manuscript was written by Li Chen, and all authors commented on previous versions of the manuscript. All authors read and approved the final manuscript.

Acknowledgements The authors would like to acknowledge all the co-workers who participated in this study.

Authors' information ${ }^{1}$.School of Humanities, Sciences \& Fine Arts囚Fujian Medical University, Fuzhou Fujian 350122, China ${ }^{2}$. School of Public Health, Fujian Medical University, Fuzhou Fujian 350122, China ${ }^{3}$. Zhangzhou Municipal Hospital of Fujian Province and Zhangzhou Affiliated Hospital of Fujian Medical University, Zhangzhou Fujian 353000, China ${ }^{4}$. Nanpin Affiliated of Fujian Medical University, Nanpin Fujian 363000, China ${ }^{5}$ Affiliated Fuzhou First Hospital of Fujian Medical University, Fuzhou Fujian 350009, China

\section{References}

1. Coronavirus disease (COVID-2019) situation reports: World Health Organization (WHO), https://www.who.int/emergencies/diseases/novel-coronavirus2019/situation-reports; [updated 10 July 2020. Situation report-172].

2. Chen X, Oshima K, Schott D, et al. Assessment of treatment response during chemoradiation therapy for pancreatic cancer based on quantitative radiomic analysis of daily CTs: An exploratory study. PLoS ONE 2017;12(6):e0178961-e178913.

3. Ai T, Yang Z, Hou H, et al. Correlation of chest CT and RT-PCR testing in coronavirus disease 2019 (COVID-19) in China: a report of 1014 cases. 2020.

4. Hosseiny M, Kooraki S, Gholamrezanezhad A, Reddy S, Myers L. Radiology Perspective of Coronavirus Disease 2019 (COVID-19): Lessons From Severe Acute Respiratory Syndrome and Middle East Respiratory Syndrome. AJR Am J Roentgenol 2020; 214(5):1078-1082.

5. Salehi S, Abedi A, Balakrishnan S, Gholamrezanezhad A. Coronavirus Disease 2019 (COVID-19): A Systematic Review of Imaging Findings in 919 Patients. AJR Am J Roentgenol 2020;215(1):87-93.

6. Bernheim A, Mei X, Huang M, et al. Chest CT Findings in Coronavirus Disease-19 (COVID-19): Relationship to Duration of Infection. Radiology. 2020; 295(3):200463-200481.

7. Gillies RJ, Kinahan PE, Hricak H. Radiomics: Images Are More than Pictures, They Are Data. Radiology 2016; 278(2):563-577.

8. Lambin P, Rios-Velazquez E, Leijenaar R, et al. Radiomics: extracting more information from medical images using advanced feature analysis. Eur $\mathrm{J}$ Cancer 2012; 48(4):441-446.

9. Aerts HJWL, Velazquez ER, Leijenaar RTH, et al. Decoding tumour phenotype by noninvasive imaging using a quantitative radiomics approach. Nat Commun 2014; 5:4006-40014.

10. Sandrasegaran K, Lin Y, Asare-Sawiri M, Taiyini T, Tann M. CT texture analysis of pancreatic cancer. Eur Radiol 2019; 29(3):1067-1073.

11. Chen X, Oshima K, Schott D, et al. Assessment of treatment response during chemoradiation therapy for pancreatic cancer based on quantitative radiomic analysis of daily CTs: An exploratory study. PLoS ONE 2017;12(6):e0178961-e0178974.

12. Chakraborty J, Langdon-Embry L, Cunanan KM, et al. Preliminary study of tumor heterogeneity in imaging predicts two year survival in pancreatic cancer patients. PLoS ONE 2017;12(12):e0188022-e018036.

13. Kim BR, Kim JH, Ahn SJ, et al. CT prediction of resectability and prognosis in patients with pancreatic ductal adenocarcinoma after neoadjuvant treatment using image findings and texture analysis. Eur Radiol 2019; 29(1):362-372.

14. Yun G, Kim YH, Lee YJ, Kim B, Hwang J-H, Choi DJ. Tumor heterogeneity of pancreas head cancer assessed by CT texture analysis: association with survival outcomes after curative resection. Sci Rep 2018;8(1):7226-7235.

15. Attiyeh MA, Chakraborty J, Doussot A, et al. Survival Prediction in Pancreatic Ductal Adenocarcinoma by Quantitative Computed Tomography Image Analysis. Ann Surg Oncol 2018; 25(4):1034-1042.

16. Han L, Zhu Y, Liu Z, et al. Radiomic nomogram for prediction of axillary lymph node metastasis in breast cancer. Eur Radiol 2019; 29(7):3820-3829.

17. Mengjie FANG, Bingxi HE, Li LI. et al. CT radiomics can help screen the coronavirus disease 2019 (COVID-19): a preliminary study. China Inf. 2020; 63:172103-172103:8.

18. Chen $\mathrm{H}$, Zeng M, Wang $\mathrm{X}$, et al. A CT- based radiomics nomogram for predicting prognosis of coronavirus disease 2019 (COVID-19) radiomics nomogram predicting COVID-19. Br J Radiol 2020; 94: 20200634.

19. Wang B, Li M, Ma H, et al. Computed tomography-based predictive nomogram for differentiating primary progressive pulmonary tuberculosis from community-acquired pneumonia in children. BMC Med Imaging 2019;19(1):63-73.

20. L.L.C. Kasun, H. Zhou, G.-B. Huang, and C. M. Vongc. “Representational learning with ELMs for big data” IEEE Intell. Syst.,2016. 28(6):31-34.

21. Cai, W.; Liu, T.; Xue, X et al. CT Quantification and Machine-learning Models for Assessment of Disease Severity and Prognosis of COVID-19 PatientsAcademic Radiology, 2020. 27(12): p. 1665-1678.

22. Xie X, Zhong Z, Zhao W, Zheng C, Wang F, Liu J. Chest CT for Typical Coronavirus Disease 2019 (COVID-19) Pneumonia: Relationship to Negative RT-PCR Testing. Radiology 2020;296(2):E41-E45.

23. Yang L, Yang J, Zhou X, et al. Development of a radiomics nomogram based on the 2D and 3D CT features to predict the survival of non-small cell lung cancer patients. Eur Radiol 2019;29(5):2196-2206. 
24. Malik J., Sainarayanan G., Dahiya R. (2011) Palmprint Authentication by Phase Congruency Features. In: Abraham A., Mauri J.L., Buford J.F., Suzuki J., Thampi S.M. (eds) Advances in Computing and Communications. ACC 2011. Communications in Computer and Information Science, vol 192. Springer, Berlin, Heidelberg.

25. Schenk V.U.B., Brady M. (2003) Improving Phase-Congruency Based Feature Detection through Automatic Scale-Selection. In: Sanfeliu A., Ruiz-Shulcloper J. (eds) Progress in Pattern Recognition, Speech and Image Analysis. CIARP 2003. Lecture Notes in Computer Science, vol 2905. Springer, Berlin, Heidelberg.

26. Peng F., Peng W., Zhang C. (2019) Evaluation of sEMG-Based Feature Extraction and Effective Classification Method for Gait Phase Detection. In: Sun F., Liu H., Hu D. (eds) Cognitive Systems and Signal Processing. ICCSIP 2018. Communications in Computer and Information Science, vol 1006. Springer, Singapore.

27. Jacanamejoy Jamioy C., Meneses-Casas N., Forero M.G. (2019) Image Feature Detection Based on Phase Congruency by Monogenic Filters with New Noise Estimation. In: Morales A., Fierrez J., Sánchez J., Ribeiro B. (eds) Pattern Recognition and Image Analysis. IbPRIA 2019. Lecture Notes in Computer Science, vol 11867. Springer, Cham.

28. Hacihaliloglu I., Abugharbieh R., Hodgson A., Rohling R. (2008) Bone Segmentation and Fracture Detection in Ultrasound Using 3D Local Phase Features. In: Metaxas D., Axel L., Fichtinger G., Székely G. (eds) Medical Image Computing and Computer-Assisted Intervention - MICCAI 2008. MICCAI 2008. Lecture Notes in Computer Science, vol 5241. Springer, Berlin, Heidelberg.

\section{Figures}
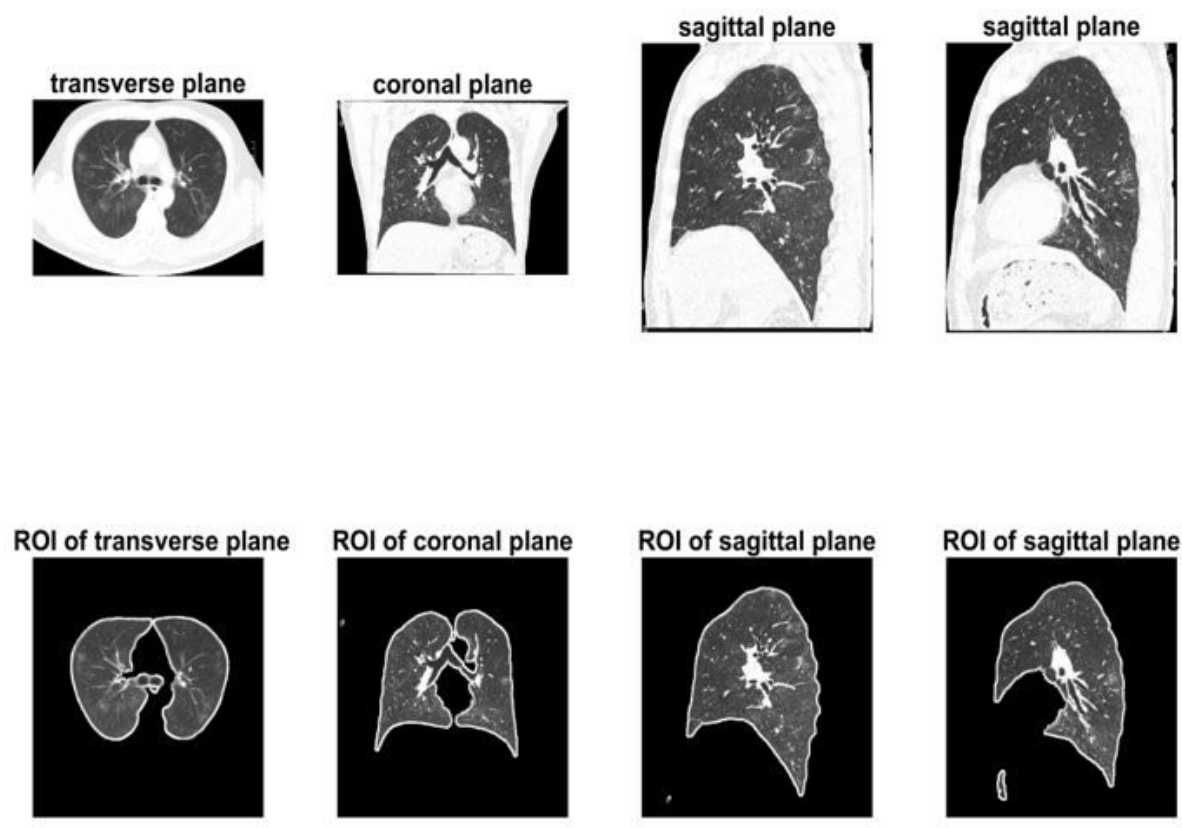

\section{Figure 1}

CT image ROI segmentation of coronal plane囚transverse plane and sagittal plane ROI segmentation results 
$\begin{array}{lllllllllllllllll}15 & 12 & 12 & 13 & 13 & 14 & 12 & 11 & 9 & 9 & 9 & 8 & 7 & 6 & 6 & 2 & 1\end{array}$

A.
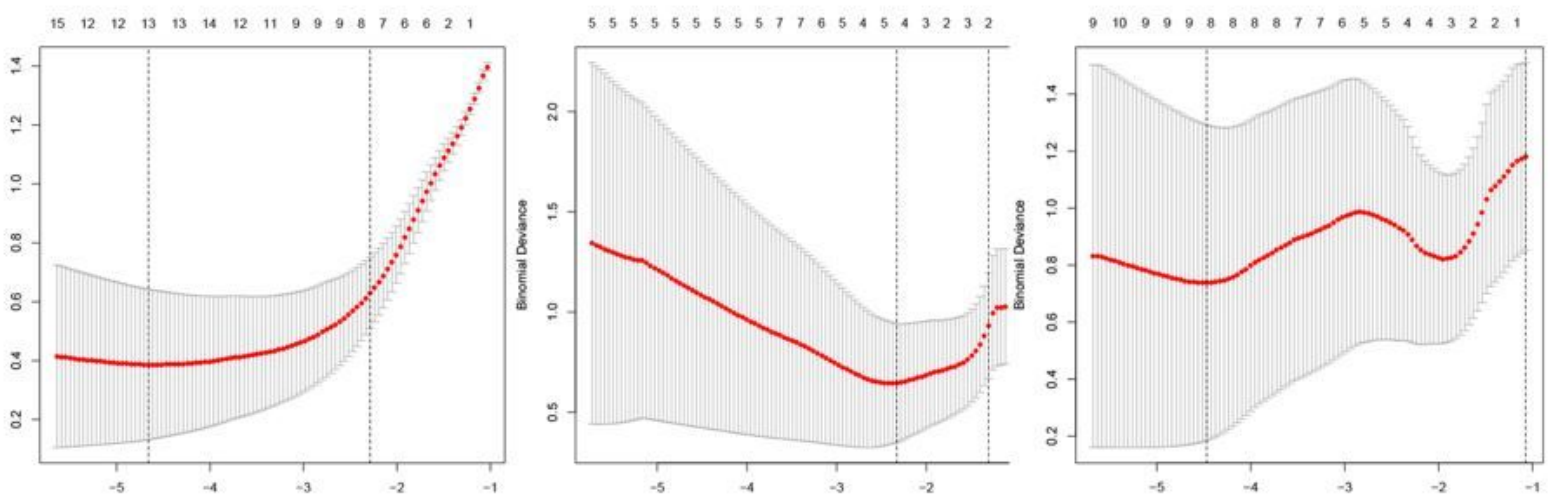

B.

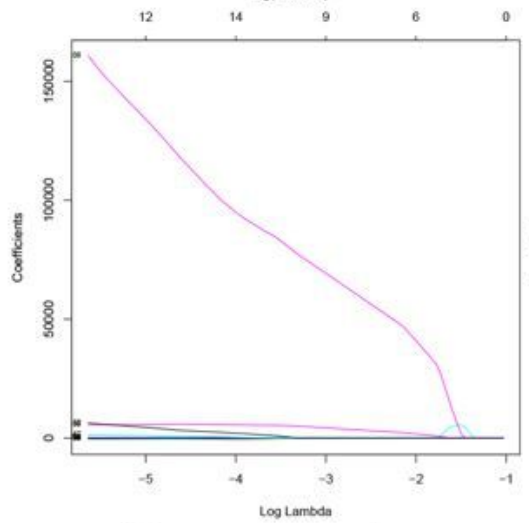

Transverse plane

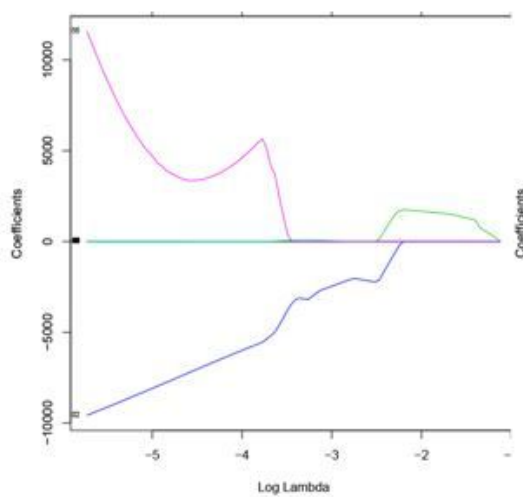

Coronal plane

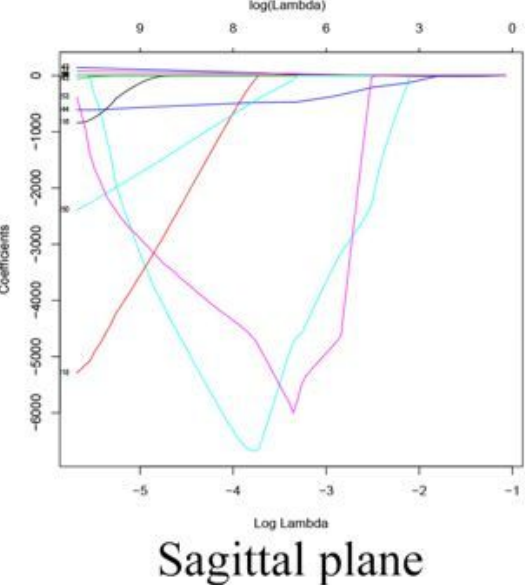

Figure 2

Selection of radiomics features using the LASSO logistic regression models with 10 -fold cross test from three different views. A. Partial likelihood deviance was plotted versus log (Lambda). The vertical dotted line indicated the lambda value with the minimum error and the largest lambda value where the deviance was within standard error of the minimum. B. LASSO coefficient profiles of the features associated with COVID-19. 

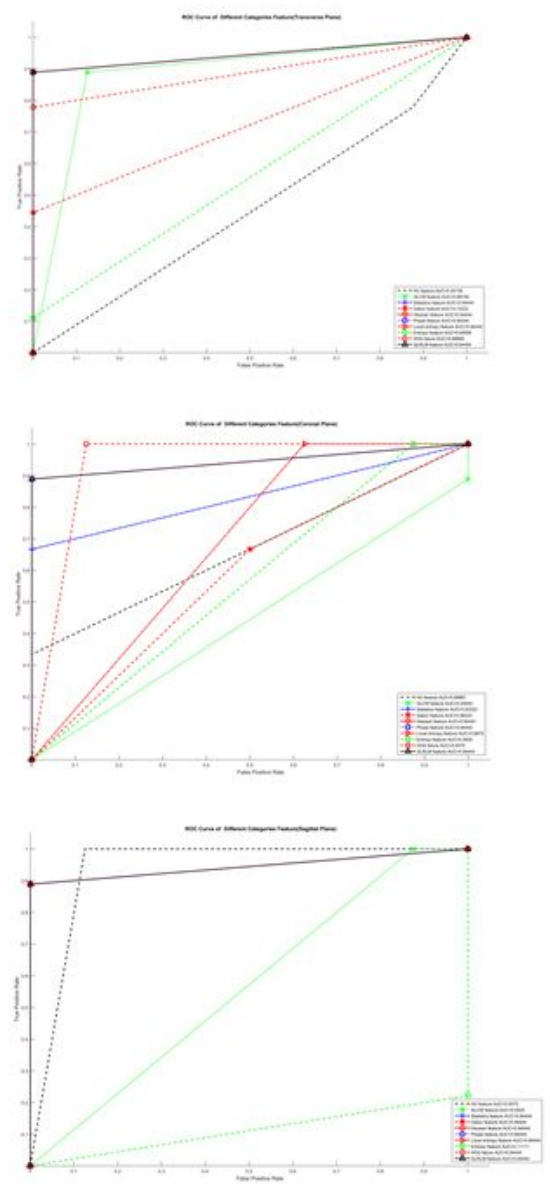

Figure 3

The ROC curves of different categories radiomics in the transverse plane, coronal plane and sagittal plane based on the PSO-DELM model Supplementary Files

This is a list of supplementary files associated with this preprint. Click to download.

- AppendixA1.docx 